\title{
BIOMASSA FITOPLANCTÔNICA (CLOROFILA A) E PARÂMETROS AMBIENTAIS NA PRAIA DE CARNE DE VACA, GOIANA, PERNAMBUCO, BRASIL
}

\author{
Maria Jaciane de Almeida CAMPELO ${ }^{1}$ \\ José Zanon de Oliveira PASSAVANTE ${ }^{2}$ \\ Maria Luise KOENING ${ }^{2}$ \\ ${ }^{1}$ Dissertação de Mestrado em Biologia Vegetal - UFPE \\ ${ }^{2}$ Departamento de Oceanografia - UFPE
}

\section{RESUMO}

A praia de Carne de Vaca, está localizada no litoral norte do Estado de Pernambuco, entre as coordenadas P34'40" e P34'57' Lat. S, 3452'38' e 3452'34" Long. W, Goiana - Pernambuco, Brasil. Com o objetivo de quantificar a biomassa fitoplanctônica (clorofila $a$ ) e correlacioná-la com os parâmetros hidrológicos e climáticos foram coletadas amostras bimestralmente na superfície da água, nos regimes de baixa-mar e preamar de um mesmo dia, no período de fevereiro a dezembro de 1998 em duas estações delimitadas para o estudo. A coleta das amostras de oxigênio dissolvido e demanda bioquímica do oxigênio (DBO) foram efetuadas com garrafa oceanográfica de Nansen e as amostras de salinidade, $\mathrm{pH}$, nutrientes (nitrato, nitrito, fosfato e silicato), foram feitas com garrafas plásticas. As amostras para o estudo da biomassa (clorofila $a$ ) foram filtradas por um sistema de filtração da marca Millipore e a determinação da clorofila $a$ foi realizada pela análise espectrofotométrica. Os parâmetros climatológicos apresentaram um nítido ciclo sazonal e os hidrológicos não apresentaram um padrão de distribuição. Os teores de biomassa (clorofila $a$ ) variaram de 0,75 a $18,94 \mathrm{mg} . \mathrm{m}^{-3}$ e não houve grandes diferenças nas estações de coletas, embora, revelando-se ligeiramente mais elevados na estação 1. Considerando-se os teores de clorofila $a$, pode-se classificar a praia de Carne de Vaca como um ambiente eutrófico, apresentando regime de salinidade variando de eualino a polialino. A estação 1, é condicionada às influências dos rios da região, apresentando níveis mais elevados de fertilidade da água e a estação 2 , recebe influência de um pequeno aporte de água doce que sustenta um manguezal localizado adjacente a esta estação.

Palavras-chave: clorofila $a$, área costeira, hidrologia

\section{ABSTRACT \\ Phytoplankton Biomass (Chlorophyll A) and Environmental Parameters of the Beach of Carne de Vaca - Goiana, Pernambuco, Brazil.}

The beach of Carne de Vaca, is located at the littoral north of Pernambuco State between $7^{\circ} 34^{\prime} 40^{\prime \prime}$ and $7^{\circ} 34^{\prime} 57^{\prime \prime} \mathrm{S}$; 34 $52^{\circ} 38^{\prime \prime}$ and $34^{\circ} 52^{\prime} 34^{\prime \prime} \mathrm{W}$, Goiana, Pernambuco, Brazil. In order to quantify the microphytoplankton biomass (chlorophyll a) and its correlation to hydrological and climatological parameters, samples were bimonthly collected at surface in two fixed stations, from February to December/1998 at diurnal low and high tide. Water for measuring dissolved oxygen and BOD were 
collected with a Nansen Bottle; and water for salinity, $\mathrm{pH}$ and nutrients (nitrate, nitrite, phosphate and silicate) were obtained with plastic bottles. Biomass samples were obtained by water filtration in a Millipore filter system and chrorophyll $\boldsymbol{a}$ was determined by spectrophotometric analysis. The climatological data presented a small seasonal cycle while hydrological data didn't presented a distribution pattern according to the dry and rainy season. Chlorophyll $\boldsymbol{a}$ varied from 0.75 to $18.94 \mathrm{mg} . \mathrm{m}-3$ and no significant differences were observed between stations, although station 1 presented higher values. According to chlorophyll $a$ values the beach of Carne de Vaca can be classified as eutrophic, with salinity varying from euhaline to polyhaline regime. Station 1 is conditioned by the rivers influence and presents higher fertility. Station 2 depends upon marine flux and from waters of the freatic shit upwelling in the area, allowing the mangrove development in the area.

Key-words: chlorophyll $a$, coastal waters, hidrology

\section{INTRODUÇÃO}

A clorofila $a$ é o tipo de pigmento fisiologicamente mais importante, presente em todos grupos vegetais (Varela \& Massa, 1981), sendo sua quantidade um bom estimador da biomassa fitoplanctônica presente nas águas naturais. Além da relação entre a clorofila $a$ e os outros pigmentos que intervém na fotossíntese e entre seus produtos de degradação, proporcionarem informações sobre o estado fisiológico do fitoplâncton e indiretamente, sobre a produção de uma população (Margalef, 1974).

Ryther et al (1956), afirma que a produtividade das águas naturais é sempre definida com relação à quantidade de matéria orgânica que elas suportam. Segundo Passavante e Feitosa (1989), os estudos sobre a variação espaço-temporal da clorofila $a$ são de grande importância ecológica, pois permitem avaliar o potencial de produção orgânica dos ecossistemas aquáticos, podendo fornecer indicações sobre a quantidade de matéria orgânica disponível aos demais níveis tróficos.

Para Tundisi (1969), as águas tropicais costeiras podem ou não apresentar um ciclo anual do fitoplâncton, dependendo da ocorrência ou não de modificações anuais em certos fatores ambientais.

Este trabalho trata do estudo da clorofila $a$ numa área costeira de Pernambuco, na praia de Carne de Vaca - Goiana, Pernambuco e sua relação com os parâmetros hidrológicos e climatológicos, bem como, a influência dos rios Goiana e São Lourenço sobre os teores de clorofila $a$.

\section{DESCRIÇÃO DA ÁREA}

As investigações realizadas neste estudo, concentraram-se na praia de Carne de

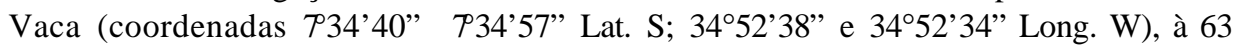
km do Recife (Fig. 1). Nesta praia desembocam dois rios, Goiana e São Lourenço, este último também denominado Megaó.

De acordo com a classificação de Köppen, a área apresenta clima considerado As' "Tropical Quente - Úmido, com chuvas de outono - inverno" (Andrade \& Lins, 1965), com dois períodos distintos no regime pluviométrico: uma estação seca ou de estiagem, que se prolonga de setembro a fevereiro (primavera-verão), e estação chuvosa, de março a agosto (outono-inverno). 
Foram demarcadas duas estações nas quais foram realizadas coletas bimestrais no período de fevereiro a dezembro de 1998 num regime de preamar e baixa-mar de um mesmo dia.

\section{CARACTERIZAÇÃO DAS ESTAÇÕES}

Estação 1 - Localizada na área costeira, recebe influência do aporte de água doce, proveniente dos rios Goiana e Megaó, e cujos valores de salinidade variaram de 21,51 a 37,68\%. Apresenta grandes depósitos de fragmentos de uma clorofícea calcária do gênero Halimeda e pequenas populações de fanerógama aquática Halodule wrightii Ascherson.

Estação 2 - Localizada a $2,5 \mathrm{~km}$ da estação 1, sendo a mesma, margeada por uma vegetação de manguezal, em que, vem sofrendo ação antrópica para fins de venda de loteamentos. Com salinidade entre 32,39 a 37,95\%o. Apresentando também populações de Halodule wrightii Ascherson.

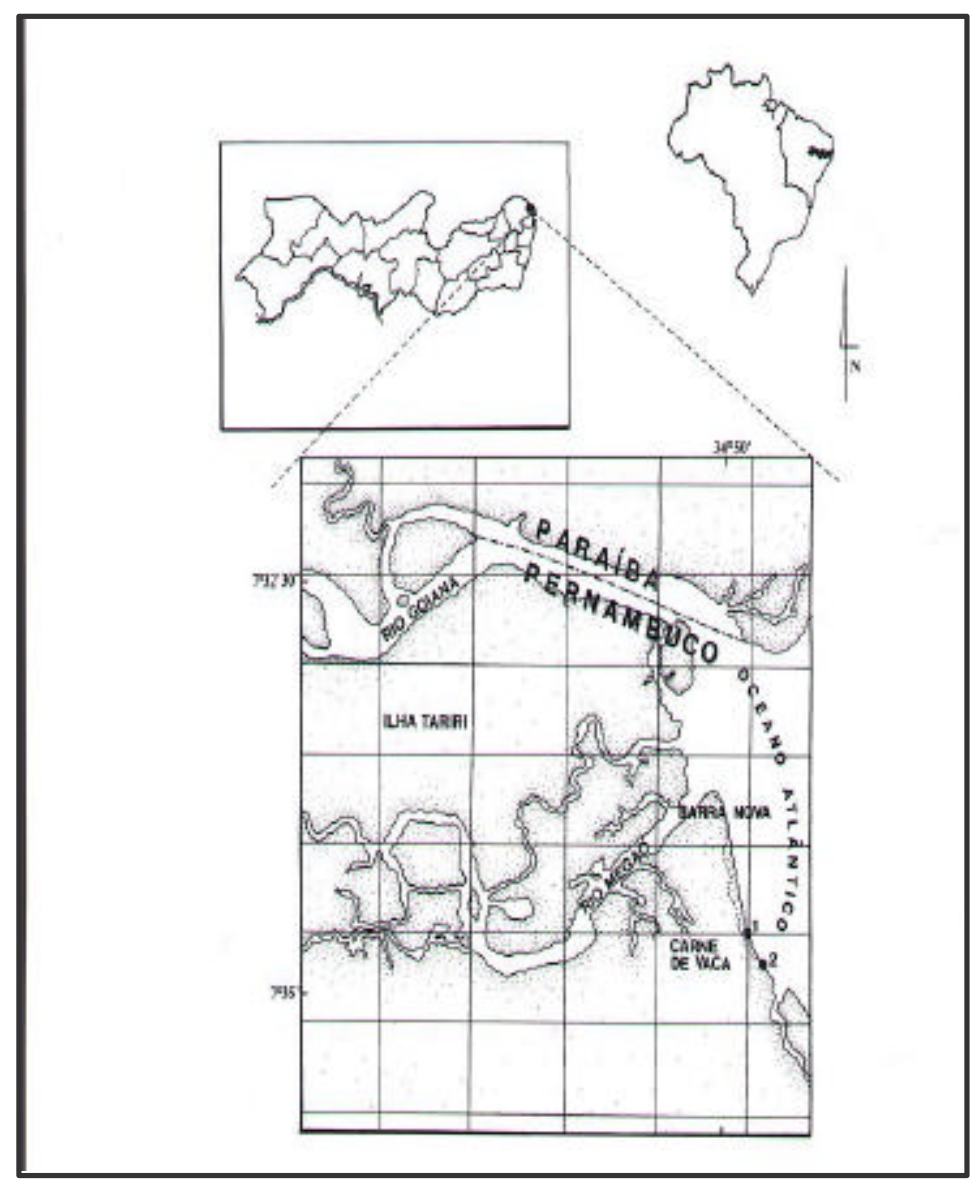

Figura 1 - Localização da área e estações de coletas. (Escala =1: 25.000) 


\section{Coleta do material}

\section{MATERIAL MÉTODOS}

Os dados climatológicos apresentados, foram cedidos pela Estação Meteorológica de Itapirema - Goiana, Pernambuco situada nos paralelos ( $7^{\circ} 34^{\prime}$ Lat. S e $35^{\circ} 00^{\prime}$ Long. W) à $14 \mathrm{~m}$ de altitude.

As coletas do material hidrológico foram realizadas através de garrafa oceanográfica de Nansen com capacidade de 1,3 litros. As análises dos sais nutrientes e do oxigênio dissolvido foram obtidas pelo método descrito por Strickland \& Parsons (1972), Grasshoff et al. (1983) e UNESCO (1973). Os valores de pH da água, foram determinados através de um potenciômetro, tipo Metronal E120 e a salinidade, pelo método de Morh-Knudsen, descrito por Strickland \& Parsons (op.cit.). A análise do DBO seguiu as recomendações descritas no Standard methods for the examination of waste water publicado pela APHA (1985). Para obter os valores dos percentuais de saturação de oxigênio dissolvido foi utilizado a International Oceanografic Tables da UNESCO (1973). SYSTAT 5.0.

A regressão linear, foi calculada utilizando-se o Programa Computacional

Para a análise da biomassa fitoplanctônica foram coletadas amostras na superfície da água e armazenadas em garrafas plásticas opacas de um litro, sendo mantidas em local livre de iluminação direta e imediatamente levadas para serem filtradas na Base Avançada da praia de Carne de Vaca do Departamento de Oceanografia da Universidade Federal de Pernambuco, onde eram filtradas pelo sistema de filtração da marca Millipore.

O método para a determinação da clorofila $a$ foi o da análise espectrofotométrica, proposta por Richards \& Thompson (1952) e modificada por Creitz \& Richards (1955).

A extração da clorofila $a$, seguiu as recomendações descritas por Parsons \& Strickland (1963) e UNESCO (1966).

As respectivas leituras de absorbâncias foram realizadas em um espectrofotômetro de marca Micronal B 280, em diferentes comprimentos de ondas (630, 645, 665 e 750nm).

Para o cálculo da concentração de clorofila $a$ aplicou-se a equação de Parsons \& Strickland (1963):

$\mathrm{mg} \cdot \mathrm{m}^{-3}=\frac{11,6 \cdot \mathrm{D}_{665}-\left(1,31 . \mathrm{D}_{645}+0,14 . \mathrm{D}_{630}+\mathrm{D}_{750)} \cdot \mathrm{v}\right.}{\mathrm{V} . \mathrm{L}}$

Onde: $\bullet \mathrm{v}=$ volume de acetona $90 \%(10 \mathrm{ml})$;

- $\mathrm{V}=$ volume da amostra filtrada (litro);

- L = caminho óptico da cubeta $(1 \mathrm{~cm})$;

- $\mathrm{D}=$ leituras da absorbâncias nos respectivos comprimentos de onda a que se referem seus índices. 


\section{RESULTADOS}

\section{Biomassa Fitoplânctônica}

$\mathrm{Na}$ estação 1 , a concentração da clorofila $a$ total na baixa-mar, variou de 1,18mg. $\mathrm{m}^{-3}$ a $4,09 \mathrm{mg} \cdot \mathrm{m}^{-3}$ em abril e fevereiro, respectivamente. Na preamar, nota-se que o valor mínimo foi de $3,78 \mathrm{mg} \cdot \mathrm{m}^{-3} \mathrm{em}$ fevereiro e máximo de $12,68 \mathrm{mg} \cdot \mathrm{m}^{-3} \mathrm{em}$ junho.

Observou-se que na estação 2 , no regime de baixa-mar a concentração de clorofila $a$ total, variou de $1,19 \mathrm{mg} \cdot \mathrm{m}^{-3}$ em fevereiro a $4,24 \mathrm{mg} \cdot \mathrm{m}^{-3}$ em dezembro. No regime de preamar, o valor mínimo foi de $0,75 \mathrm{mg} \cdot \mathrm{m}^{-3}$ em abril e máximo de 18,94 mg.m $\mathrm{m}^{-3}$ em junho.

Analisando ambas estações e os diferentes regimes de marés, nota-se que de uma maneira geral, a concentração da clorofila $a$ foi mais elevada durante o regime de preamar com uma média de $7,35 \mathrm{mg} \cdot \mathrm{m}^{-3}$ na estação 1 e $7,18 \mathrm{mg} \cdot \mathrm{m}^{-3}$ na estação 2 , com concentrações mais elevadas durante o período chuvoso (Fig. 2).

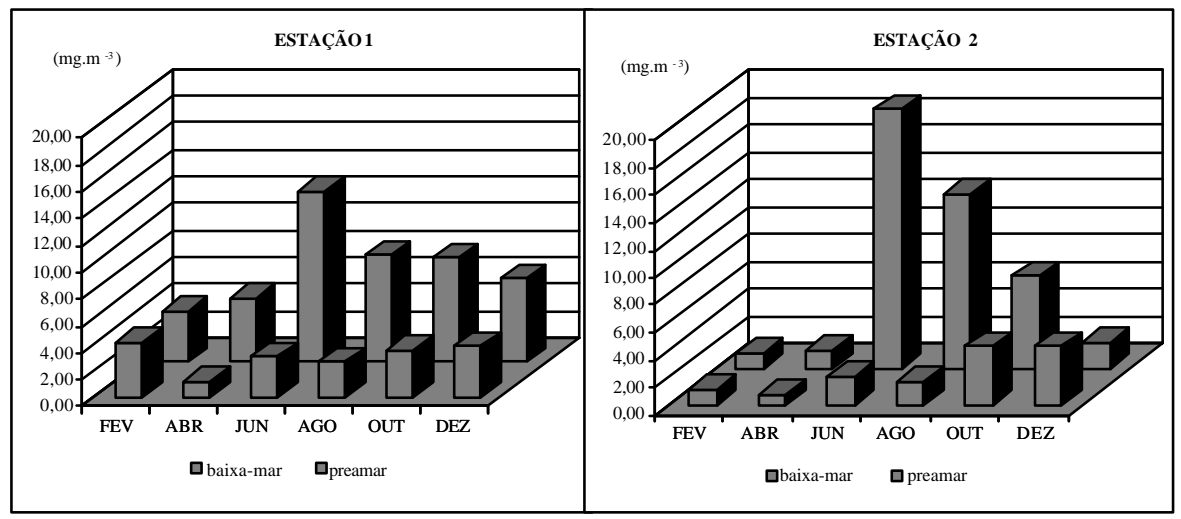

Figura 2- Variação da clorofila $a$ nos regimes de baixa-mar e preamar da praia de Carne de Vaca, Goiana Pernambuco durante o ano de 1998.

\section{Climatologia}

Os dados climatológicos fornecidos pela estação meteorológica de Itapirema Goiana - Pernambuco, demonstraram que em geral, ocorreu um nítido ciclo sazonal durante o período de coleta na praia de Carne de Vaca (Tab. 1).

Os valores da temperatura do ar, apresentaram oscilações ao longo do ano. A temperatura mínima registrada foi de $20,9^{\circ} \mathrm{C}$ em agosto e máxima de $30,5^{\circ} \mathrm{C}$ em fevereiro, sendo a amplitude térmica anual de $9,6^{\circ} \mathrm{C}$. Nota-se que a temperatura diminuiu a partir de abril, atingindo valor mínimo no mês de agosto, quando ocorreu a maior precipitação do ano. 
Os valores de precipitação pluviométrica apresentaram grandes variações, com valor mínimo de 19,4mm em dezembro e máximo de 239,1mm em agosto. A precipitação média no ano foi de $88,5 \mathrm{~mm}$.

A insolação total não revelou variações acentuadas. Apresentou índices mínimo e máximo em outubro e dezembro, cujos valores foram 203,3h e 310,3h, respectivamente. A média da insolação foi de 216,9/h.

A umidade relativa do ar, apresentou valor mínimo às $9 \mathrm{~h}$ de $71 \%$ em dezembro e máximo de $87 \%$ em agosto, verificou-se que neste horário, a umidade relativa tende a aumentar a partir de abril. Já às $15 \mathrm{~h}$ foram registrados valores mínimo e máximo de $70 \%$ em dezembro, coincidindo com o período de estiagem e de $85 \%$ em junho.

De uma maneira geral, as variações da umidade relativa foram mais acentuadas no período chuvoso.

\section{Hidrologia}

\section{Estação 1}

A salinidade na baixa-mar, apresentou valor mínimo de 18,96\%o em outubro e máximo de 36,74\%o em fevereiro. Já na preamar, o valor mínimo foi de 18,81\%o, também registrado em outubro e máximo de 37,68\%o em dezembro.

$\mathrm{O}$ oxigênio dissolvido, em regime de baixa-mar, apresentou valor mínimo de $4,41 \mathrm{ml} \mathrm{L}^{-1}$ em fevereiro e máximo de $5,95 \mathrm{ml} \mathrm{L}^{-1}$ em outubro, que corresponde a um percentual de saturação de oxigênio de $102,08 \%$ e $121,18 \%$, respectivamente. Na preamar, estes resultados variaram um pouco durante o período de estudo, revelando valor mínimo de $3,44 \mathrm{ml} \mathrm{L}^{-1}$ em junho, que corresponde ao um percentual de saturação de oxigênio de $71,22 \%$ e valor máximo de $6,06 \mathrm{ml} \mathrm{L}^{-1}$ em agosto, que corresponde a um percentual de saturação de oxigênio de $126,25 \%$.

A demanda bioquímica do oxigênio (DBO) na baixa-mar, apresentou valor mínimo de $0,12 \mathrm{mg} \cdot \mathrm{L}^{-1}$ em outubro e máximo de $0,73 \mathrm{mg} . \mathrm{L}^{-1}$ em fevereiro. Na preamar, o menor valor foi de $0,17 \mathrm{mg} . \mathrm{L}^{-1}$ ocorrido em fevereiro e o maior valor foi de $1,38 \mathrm{mg} . \mathrm{L}^{-1}$ em agosto.

Os valores de $\mathrm{pH}$ na baixa-mar, variaram de 7,85 a 8,38 entre os meses de dezembro e fevereiro, respectivamente. $\mathrm{Na}$ preamar, foram mantidos valores semelhantes, com mínimo registrado de 7,89 em outubro e máximo de 8,47 em agosto.

Os valores de nitrito $\left(\mathrm{NO}_{2}-\mathrm{N}\right)$ na baixa-mar, foram de $0,001 \mu \mathrm{mol} . \mathrm{L}^{-1}$ em junho e de $0,246 \mu$ mol. $L^{-1}$ em fevereiro. Na preamar, os teores mínimo e máximo foram de $0,010 \mu \mathrm{mol} . \mathrm{L}^{-1}$ em outubro e de $0,328 \mu \mathrm{mol} . \mathrm{L}^{-1}$ em agosto, respectivamente.

Os teores de nitrato $\left(\mathrm{NO}_{3}-\mathrm{N}\right)$ na baixa-mar, foram de $0,454 \mu \mathrm{mol} \cdot \mathrm{L}^{-1} \mathrm{em}$ fevereiro e de $1,756 \mu m o l . L^{-1}$ em outubro. Na preamar, também ocorreu uma variação acentuada desses valores, apresentando em fevereiro valor mínimo de $0,696 \mu \mathrm{mol} . \mathrm{L}^{-1} \mathrm{e}$ em agosto valor máximo de $1,857 \mu \mathrm{mol} . \mathrm{L}^{-1}$.

Os teores de fosfato $\left(\mathrm{PO}_{4}-\mathrm{P}\right)$, em regime de baixa-mar, revelaram um mínimo de $0,216 \mu$ mol. $\mathrm{L}^{-1}$ em junho e máximo de $0,441 \mu \mathrm{mol} . \mathrm{L}^{-1} \mathrm{em}$ abril. Na preamar, foi registrado valor mínimo de $0,001 \mu \mathrm{mol} . \mathrm{L}^{-1}$ em outubro e máximo de $0,424 \mu \mathrm{mol} . \mathrm{L}^{-1} \mathrm{em}$ junho.

Os teores de silicato $\left(\mathrm{SiO}_{2}-\mathrm{Si}\right)$, revelaram grandes oscilações no período de coleta, apresentando na baixa-mar mínimo de $1,479 \mu \mathrm{mol} . \mathrm{L}^{-1}$ em outubro e dezembro, 
respectivamente e máximo de $45,422 \mu$ mol. $\mathrm{L}^{-1}$ em agosto. Na preamar, o valor mínimo foi de $1,056 \mu \mathrm{mol} . \mathrm{L}^{-1}$ em outubro e o valor máximo foi de $76,139 \mu \mathrm{mol} . \mathrm{L}^{-1}$ em agosto

\section{Estação 2}

Em regime de baixa-mar, o valor mínimo de salinidade foi de 19,25\% em outubro e máximo de 36,89\%o em dezembro. Em regime de preamar, o valor mínimo foi de 19,84\%o também registrado em outubro e máximo de 37,95\% em dezembro .

$\mathrm{O}$ oxigênio dissolvido na baixa-mar, apresentou valor mínimo de $3,86 \mathrm{ml} \mathrm{L}^{-1}$ em junho e o valor máximo de $5,53 \mathrm{ml} \mathrm{L}^{-1}$ em outubro, que corresponde respectivamente a um percentual de saturação de oxigênio de $86,16 \%$ e $108,85 \%$. Em regime de preamar, tais valores variaram de $2,44 \mathrm{ml} \mathrm{L}^{-1}$ em junho, que corresponde a um percentual de saturação de $50,83 \%$ e máximo de $5,53 \mathrm{ml} \mathrm{L}^{-1}$ em dezembro, correspondendo $139,62 \%$ de saturação de oxigênio.

A demanda bioquímica de oxigênio em regime baixa-mar, revelou valor mínimo de $0,10 \mathrm{mg} . \mathrm{L}^{-1}$ em dezembro e máximo de $1,47 \mathrm{mg} . \mathrm{L}^{-1}$ em outubro. Na preamar, o valor ficou entre 0,18 a $7,28 \mathrm{mg}^{-\mathrm{L}^{-1}}$ em fevereiro e agosto, respectivamente.

$\mathrm{O}$ pH na baixa-mar, foi de 7,90 e 8,38 em dezembro e junho, respectivamente. $\mathrm{Na}$ preamar, foram obtidos valores de 7,93 e 8,50 em dezembro e fevereiro, respectiv amente.

Os valores de $\left(\mathrm{NO}_{2}-\mathrm{N}\right)$ na baixa-mar, foram de $0,001 \mu \mathrm{mol} . \mathrm{L}^{-1}$ nos meses de junho a dezembro e de $0,070 \mu \mathrm{mol} . \mathrm{L}^{-1}$ em abril. Na preamar, estes teores apresentaram valor mínimo semelhante ao da baixa-mar nos meses de outubro e dezembro e máximo de $0,072 \mu \mathrm{mol} . \mathrm{L}^{-1}$ nos meses de abril e junho, respectivamente. Nota-se que há uma diminuição das concentrações de nitrito no período de junho a dezembro em ambas estações.

Os valores de $\left(\mathrm{NO}_{3}-\mathrm{N}\right)$ na baixa-mar, revelaram um mínimo de $0,125 \mu$ mol. $\mathrm{L}^{-1}$ em outubro e um máximo de $0,969 \mu \mathrm{mol}_{\mathrm{L}} \mathrm{L}^{-1}$ em abril. Na preamar, os valores mínimo e máximo foram de $0,567 \mu$ mol. $\mathrm{L}^{-1}$ e $1,453 \mu \mathrm{mol} . \mathrm{L}^{-1}$ em agosto e dezembro, respectivamente.

Os teores de $\left(\mathrm{PO}_{4}-\mathrm{P}\right)$ apresentaram na baixa-mar, valor mínimo de $0,062 \mu \mathrm{mol} . \mathrm{L}^{-1}$ em agosto e máximo de $0,241 \mu \mathrm{mol} . \mathrm{L}^{-1} \mathrm{em}$ abril. Na preamar, o valor mínimo foi de $0,066 \mu m o l . L^{-1}$ em outubro e o valor máximo foi de $0,678 \mu \mathrm{mol} . \mathrm{L}^{-1} \mathrm{em}$ junho.

Os teores de $\left(\mathrm{SiO}_{2}-\mathrm{Si}\right)$ na baixa-mar, variaram entre $1,225 \mu \mathrm{mol} . \mathrm{L}^{-1} \mathrm{em}$ dezembro e $57,802 \mu \mathrm{mol} . \mathrm{L}^{-1}$ em fevereiro. Na preamar, o mínimo foi de $1,014 \mu \mathrm{mol} . \mathrm{L}^{-1}$

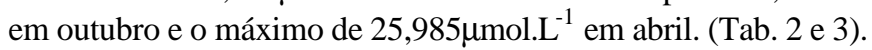

\section{Regressão linear múltipla}

A partir da análise de regressão linear múltipla realizada com os parâmetros hidrológicos, foi possível obter uma correlação entre a clorofila $a$, a salinidade e o fosfato. A salinidade apresentou uma relação inversa com à clorofila $a$, revelando que quanto menor a salinidade, maior os índices de clorofila $a$ e quanto maior o índice de fosfato, maior a concentração de clorofila $a$. Essa relação mostrou-se significativa, com regressão linear múltipla de $\mathrm{R}^{2}=0,474 ; \mathrm{F}=9,469$ e $\mathrm{P}=0,001$. 


\section{DISCUSSÃO}

Os valores de clorofila $a$ na praia de Carne de Vaca, mostraram-se mais levados na estação 1, em ambos regimes de marés. Na preamar, estas concentrações, revelaram-se maiores nos meses em que foram registrados precipitação pluviométrica. Na baixa-mar, as concentrações de clorofila $a$ foram bastantes oscilantes.

O mesmo foi observado por Munõz-Anderson \& Millan-Nuñez (1991), que encontraram no Estero de Punta Banda, na Baixa Califórnia valores sempre mais elevados na preamar.

Para Sassi \& Kutner (1982), os teores de clorofila $a$, no Saco da Ribeira (Ubatuba, SP), estão associados às concentrações de nutrientes no ambiente, onde os valores máximos foram registrados no período chuvoso em consequiência do maior aporte destes elementos provocados pela drenagem terrestre.

Passavante (1981), também encontrou no Canal de Santa Cruz, picos máximos de clorofila $a$ coincidindo com picos máximos de precipitação na região. Ressurreição (1990), afirma que a precipitação pluviométrica contribui como fator de inibição da concentração de clorofila $a$ nas estações próximas da costa, enquanto que nas estações mais afastadas verifica-se uma correção positiva entre precipitação e biomassa do fitoplâncton, com uma contribuição das chuvas para o incremento de teor de clorofila $a$.

O mesmo não foi observado por Teixeira \& Gaeta (1991), que perceberam que a amplitude da variação da temperatura da água tem provável influência sobre o aumento ou decréscimo da clorofila $a$, e assim, em temperaturas mais elevadas e conseqüentemente menos chuvas, o conteúdo da clorofila $a$ variou de 1,48 a 4,80mg.m ${ }^{3}$, mostrando-se elevado em relação aos meses com chuvas.

Já Feitosa (1997), encontrou para o sistema estuarino dos rios Goiana e São Lourenço, teores de clorofila $a$ mais elevados durante o inverno em ambos regimes de marés, obtendo-se valor mínimo de $6,47 \mathrm{mg} \cdot \mathrm{m}^{-3}$ e máximo de $11,75 \mathrm{mg} \cdot \mathrm{m}^{-3}$.

Brandini (1986), enfatiza que as concentrações de clorofila $a$ na superfície durante o inverno sofrem variações mais intensas ao longo da costa, enquanto que no oceano essas variações são menos pronunciadas com valores inferiores aos obtidos para a costa.

De uma maneira geral, pode-se dizer que os teores de clorofila $a$, se enquadram nos padrões para as áreas costeiras, e são comparáveis aos trabalhos realizados por Passavante et al. (1987-1989), na costa de Pernambuco em frente à Ilha de ItamaracáPE, que registraram para clorofila $a$, um valor máximo de $13,50 \mathrm{mg} / \mathrm{m}^{3}$ e mínimo de $0,13 \mathrm{mg} / \mathrm{m}^{3}$ e Ressurreição et al. (1996), registraram uma biomassa entre 0,05 a $32,29 \mathrm{mg} / \mathrm{m}^{3}$, em estudo realizado num perfil perpendicular à costa, em frente ao Porto do Recife.

Considerando os valores de biomassa para a praia de Carne de Vaca, a área foi classificada como eutrófica.

Passavante \& Koening (1984), relatam que no estuário do rio Botafogo, os valores de biomassa variaram de 1,6 a $19,0 \mathrm{mg} / \mathrm{m}^{-3}$ e estes valores demonstram que esta região é bastante eutrófica, com possibilidade de sustentar uma elevada produção secundária. 
Considera-se que na praia de Carne de Vaca, o processo de enriquecimento das águas, a partir do fluxo de águas fluviais, parece ser o fator mais importante para o aumento da biomassa (clorofila $a$ ), bem como, a ressuspensão do sedimento durante a preamar.

De um modo geral, pode-se observar a influência dos rios da região na ecologia da estação 1, possibilitando em níveis mais elevados de fertilidade da água. Enquanto, que estação 2, além de recebe o aporte de águas marinhas, tem influência da vegetação de manguezal adjacente a mesma.

Quanto aos parâmetros climatológicos da área em estudo, pode-se observar um ciclo sazonal com períodos chuvoso e de estiagem, apesar de ter sido um ano de pouca chuva, com $40 \%$ abaixo da média, em decorrência do fenômeno El-Niño que causa por exemplo, flutuações nos padrões normais do sistema oceano-atmosfera. Podendo afetar de forma até catastrófica as condições climáticas globais, causando efeitos diversos (Ricklefs, 1996).

No período chuvoso (março a agosto), os dados de precipitação pluviométrica e umidade relativa revelaram-se mais elevados, enquanto que, os dados correspondentes a temperatura do ar e insolação total foram mais baixos. Estes parâmetros em geral, apresentaram-se no período de estiagem (setembro a fevereiro) de forma inversa.

Moura \& Passavante (1994-95), estudando a baía de Tamandaré - Rio Formoso, Pernambuco, também relatam sobre as irregularidades climatológicas ocorridas no período de coletas, para estes autores, a falta de chuvas na região apresenta efeitos diversos sobre a biomassa (clorofila $a$ ).

Os efeitos da ausência de precipitação pluviométrica sobre os valores da clorofila $a$, também foram mencionados por Sassi (1991) e Feitosa (1988). Para estes autores, os parâmetros climatológicos e hidrológicos influem consideravelmente na clorofila $a$.

Já os parâmetros hidrológicos na área de estudo, não apresentaram um ciclo sazonal definido. Tendo sido classificada a área com base no regime de salinidade em um ambiente eualino a polialino. Já Feitosa (1997), relata que no estuário do rio Goiana - Pernambuco, os parâmetros hidrológicos apresentaram sazonalidade e os mesmos, revelaram que o sistema estuarino variou de limnético a eualino, sendo no período chuvoso, considerado como um estuário do tipo homogêneo.

No Canal de Santa Cruz, Flores-Montes et al. (1998), também enfatizam haver um padrão de sazonalidade, para os autores, no período de estiagem, os valores estáveis de salinidade, apresentaram regime salino, eualino.

Os teores de oxigênio dissolvido, na praia de Carne de Vaca, mostram-se mais elevados no período de estiagem e em regime de preamar de ambas estações. Em geral, revelando-se supersaturados, ou seja, acima de 100\%. Segundo Travassos (1991), a taxa de saturação acima de $100 \%$ encontradas no Rio Capibaribe, Recife pode ser resultado da grande atividade fotossintética dos organismos fitoplanctônicos coincidindo com o período de maior biomassa na região.

A supersaturação de oxigênio também foi observada por Teixeira \& Gaeta (1991), na Enseada do Flamengo - Ubatuba (SP), para os autores, esta saturação foi nitidamente maiores no verão, devido também, a provável maior atividade biológica dos organismos. 
Analisando os valores da demanda bioquímica do oxigênio na área de estudo, pode-se dizer, que os mesmos sofreram variações ao longo do ano, em geral, mostrando-se baixos. Os resultados obtidos indicaram que a área pode ser enquadra como não poluída.

Para Macedo \& Costa (1990), a demanda bioquímica do oxigênio constitui o índice mais importante para avaliação dos efeitos que serão produzidos sobre os organismos aquáticos, pela introdução de cargas poluidoras orgânicas.

Com relação ao $\mathrm{pH}$, este manteve-se sempre alcalino durante todo período estudado. Para Cabo (1983), o pH depende da temperatura, salinidade e pressão parcial do dióxido de carbono na atmosfera.

$\mathrm{Na}$ praia de Carne de Vaca, observou-se que os sais nutrientes foram mais acentuados na estação 1, devido ao aporte de água doce oriunda dos rios Goiana e São Lourenço.

Flores-Montes et al. (1998), estudando o Canal de Santa Cruz, revelaram que as concentrações de nitrito e nitrato foram mais elevadas no período chuvoso e as de fosfato e silicato, no período de estiagem. Para o autor, isto indica que existem outros processos, além do aporte continental que controlam a disponibilidade destes sais.

Os teores de fosfato para a área, foram considerados baixos, com pouca variação. Foi evidenciado através da análise de regressão linear múltipla, que quanto maior o índice de fosfato maior a concentração de clorofila $a$. Esta relação, mostrou-se com regressão linear múltipla, considerada significativa. Collos et al. (1997), em estudo na laguna Thau na costa do Mediterrâneo, obtiveram uma correlação significativa entre a clorofila $a$ e o nitrogênio, obtendo-se $\mathrm{R}^{2}=0,885$.

Moura \& Passavante (1994-95), também encontraram relação significativa entre os nutrientes inorgânicos e clorofila $a$, a partir da análise de regressão linear múltipla e foi confirmado, que a concentração de nutrientes é fator limitante e condicionante para o desenvolvimento fitoplanctônico em Tamandaré, Pernambuco.

Em trabalho realizado no Canal de Santa Cruz, Cavalcanti et al. (1981), mencionam que os teores de clorofila $a$ mostraram uma distribuição instável e sem um ciclo sazonal definido, sendo observado que quanto maior o teor de nitrato, maior mostrou-se o teor de clorofila $a$. Este fato, confirmou a hipótese de Macedo (1974), em que o nitrato é provavelmente considerado o fator limitante da biomassa no Canal de Santa Cruz.

O teor de fosfato na área, revelou-se como o fator que possibilita a produção de clorofila a pelo fitoplâncton. Para Cavalcanti et al. (op. cit.) a possibilidade do fósforo constituir-se como fator limitante primário nos oceanos pode ser considerada rara. Para alguns autores, o fósforo é o nutriente que controla o crescimento do fitoplâncton.

Teixeira \& Tundisi (1981), em trabalho realizado na região de Ubatuba, São Paulo, relatam que a adição de somente nitrogênio estimula o crescimento do fitoplâncton em termos de clorofila $a$ enquanto que a adição somente de fósforo não resulta em diferentes crescimento, já a adição de nitrogênio e fósforo pode produzir um acentuado aumento da clorofila $a$. Aidar et al. (1993), também mencionam sobre a limitação do nitrogênio para a biomassa fitoplanctônica na plataforma continental de Ubatuba, São Paulo. 


\section{REFFERÊNCIAS BIBLIOGRÁFICAS}

AIDAR, E., GAETA, S. M. F., GIANESELLA-GALVÃO, S. M. F., KUTNER, M. B. B., TEXEIRA, C. Ecossistema costeiro subtropical: nutrientes dissolvidos, fitoplâncton e clorofila- $a$ e suas relações com as condições oceanográficas na região de Ubatuba, SP. Boletin do Instituto Oceanográfico, São Paulo, v. 10, p. 9-43. 1993. (Publicação Especial).

AMERICAN PUBLIC HEALT ASSOCIATION (APHA). Standard methods for the examination of water and wastewater. $16 \mathrm{ed}$. New York, $1985.1268 \mathrm{p}$.

ANDRADE, G. O., LINS, R. C. Introdução a morfoclimatologia do nordeste do

Brasil. Arq. Inst. Ciência Terra, Recife, v. 4, n. 3, p. 17-28. 1965.

BRANDINI, F.P. Hidrologia e características do fitoplâncton da região sudeste do Brasil: Produção primária, biomassa, composição. 1986, 51 p. Tese (Doutorado em Ciências), Instituto Oceanográfico, Universidade de São Paulo, 1986.

CABO, F. L. Oceanografia, Biologia Marina y Pesca. Madrid, 1983, 445 p.

CAVALCANTI, L. B., MACEDO, S. J., PASSAVANTE, J. Z. O. Estudo ecológico da região de Itamaracá, Pernambuco-Brasil. XXI- Caracterização do Canal de Santa Cruz em função dos parâmentros físicos-químicos e pigmentos fotossintéticos. Trabalhos Oceanográficos da Universidade Federal de Pernambuco, Recife, v. 16, p. 157-226. 1981.

COLLOS, Y. VAQUER, A., BIBEENT, B. Variability in nitrate uptake kinecs of phytoplankton comunities in mediterranean costal lagoon. Estuarine, Coastal and Shelf Science, London, v. 44, p. 369-375. 1997.

CREITZ, G. I., RICHARDS, F. A. The estimation and caracterization of the plankton populations by pigments anallysis. III. A note on the use of millipore membrane filters in the estimation of plankton pigments. Journal of Marine Research, New Haven, v. 14, n. 3, p. 211-216. 1955.

FEITOSA, F. A. do N. Produção primária do fitoplâncton correlacionada com parâmetros bióticos na Bacia do Pina (Recife-PE). 1988. 279 p. Dissertação (Mestrado em Oceanografia Bológica) - Universidade Federal de Pernambuco, 1988.

Estrutura e produtividade da comunidade fitoplanctônica correlacionadas com parâmetros abióticos no sistema estuarino do rio Goiana (Pernambuco - Brasil). São Paulo, 1997, 250 p. Tese (Doutorado em Ciências), Instituto Oceanográfico - Universidade de São Paulo, 1997.

FLORES-MONTES, M. J., MACÊDO, S. J., KOENING, M. L., CORREIA I. L. Variação nictemeral do fitoplâncton e elementos nutrientes no Canal de Santa Cruz, Itamaracá, Brasil. Trabalhos Oceanográficos da Universidade Federal de Pernambuco, Recife, v. 26, n.1, p. 13-26.1998.

GRASSHOF, K., EHRARDT, M., KREMLING, K. Methods of seawater analysis. 2. Ed. New York: Verlag Chemie, 1983, 317 p.

MACÊDO, S. J de. Fisioecologia de alguns estuários do Canal de Santa Cruz, Itamaracá-PE). São Paulo, 1974, 121 p. Dissertação (Mestrado em Fisiologia Geral). Departamento de Fisiologia Geral do Instituto de Biociências, Universidade de São Paulo, 1974.

MACÊDO, S. J de, COSTA, K. M. P. da. Condições hidrológicas do estuário do rio Igarassu - Itamaracá - PE. Trabalhos Oceanográficos da Universidade Federal 
de Pernambuco, Recife, v. 21, p. 7-32. 1990.

MARGALEF, R. Ecologia. Barcelona: Ômega, 1974, 941 p.

MOURA, R. T., PASSAVANTE, J. Z. de O. Biomassa fitoplanctônica de Tamandaré, Rio Formoso - Pernambuco, Brasil. Trabalhos Oceanográficos da Universidade Federal de Pernambuco, Recife, v. 23, p. 1-15. 1994-95.

MUNÕZ-ANDERSON, M. A., MILLÁN-NÚÑEZ, R. Annual variation of phytoplankton productivity in estero de Punta Banda, México. Ciências Marinas, México, v. 17, n. 2, p. 57-70. 1991.

PARSONS, T. R., STRICKLAND, J. D. H. Discussion of spectrophotometric determination of marine plankton pigments, with revised equations of as certaning chlorophyll $a$ and carotenoids. Journal of Marine Research, New Raven, v.21, n.3, p. 155-163. 1963.

PASSAVANTE, J. Z. de O. Estudo ecológico da região de Itamaracá, PernambucoBrasil. XXI. Biomassa do nano e microfitoplâncton do Canal Santa Cruz. Trabalhos Oceanográficos da Universidade Federal de Pernambuco. Recife, v. 16, p. 105-156. 1981.

PASSAVANTE, J. Z. de O., GOMES, N. A., ESKINAZI-LÉÇA, E., FEITOSA, F.A.N. Variação da clorofila $a$ do fitoplâncton na plataforma continental de Pernambuco. Trabalhos Oceanográficos da Universidade Federal de Pernambuco. Recife, v. 20, p. 145-156, 1987 - 89.

PASSAVANTE, J. Z. de O., FEITOSA, F. A. do N. Hidrologia e plâncton da plataforma continental de Pernambuco. 2.Biomassa primária fitoplâncton. In: ENCONTRO BRASILEIRO DE GERENCIAMENTO COSTEIRO， 3， 1989, Fortaleza. Anais ... Fortaleza. p.373-372. 1989.

PASSAVANTE, J. Z. de O., KOENING, M. L. Estudo ecológico da região de Itamaracá, Pernambuco, Brasil. XXVI. Clorofila $a$ e material em suspensão no estuário do rio Botafogo. Trabalhos Oceanográficos da Universidade Federal de Pernambuco. Recife, v. 18, p. 207-230. 1984.

RESSURREIÇÃO, M. G. Variação anual da biomassa fitoplanctônica na plataforma continental de Pernambuco: perfil em frente ao Porto da cidade do Recife (08 ${ }^{0}$ 03' 38" Lat.S; 34 ${ }^{0}$ 42' 28" a 34" 52' 00" Long.W). Recife, 1990, 306p. Dissertação (Mestrado em Oceanografia Biológica). Universidade Federal de Pernambuco, 1990.

RESSURREIÇÃO, M. G, PASSAVANTE, J. Z. de O., MACÊDO, S. J. Estudo da plataforma continental de área do Recife (Brasil): Variação sazonal da biomassa fitoplanctônica (08 03'38" Lat. S; 34'52'28" Long. W). Trabalhos Oceanográficos da Universidade Federal de Pernambuco, Recife, v. 24, p. 3959. 1996.

RICHARDS, F. A., THOMPSON, T. G. The estimation and characterization of plankton populations by pigments analysis. II. A spectrophotometric method for the estimation of plankton pigments. Journal of Marine Research, New Haven, v.11, n.2, p.156-172. 1952.

RICKLEFS, R. A economia da natureza. 3. ed., Guanabara Koogan, 1996, 470 p.

RYTHER, J. H., MENZEL, D. W., CORWIN, N. Influence of the amazon river outflow on the ecology of the wastern tropical atlantic. I-Hydrography and nutrient chemistry. Journal Marine Research, New Haven, v. 25, n. 1, p. 69-83. 1956. 
SASSI, R., KUTNER, M .B. B. Variação sazonal do fitoplâncton da região de Saco Ribeira (Lat. 2330' S, Long. 4507'W). Ubatuba, Brasil. Boletim do Instituto Oceanográfico de São Paulo, São Paulo, v. 31, n.2 , p. 29-42. 1982.

SASSI, R. Phytoplankton and envirionmental factors in the Paraíba do Norte River Estuary, northeastern Brazil: Composition, distrubuition and quantitative remarks. Boletim do Instituto Oceanográfico de São Paulo, São Paulo, v. 39, n. 2, p. 93$115,1991$.

STRICKLAND, J. D. H., PARSONS, T. S. A. Pratical handbook of seawater analysis. , Ottawa: Bulletim Fisheries Research Board of Canada, Helsingor, v. 167, p. 1311. 1972.

TEIXEIRA, C. TUNDISI, J. G. The effects of nitrogen and phosphorus enrichements on phytoplankton in the region of Ubatuba (Lat. 2330'S - long.4506'W), Brazil. Boletim do Instituto Oceanográfico de São Paulo. São Paulo, v. 30, n. 1, p. $77-$ 86. 1981.

TEIXEIRA, C., GAETA, S. A. Variação nictemeral da clorofila $a$, produção primária do fitoplâncton e fatores ambientais da região de Ubatuba (Lat. 23 30 'S Long. $\left.45^{0} 06^{\prime}\right)$. Boletim do Instituto Oceanográfico de São Paulo. São Paulo, v. 39, n.1, p. 15-24. 1991.

TRAVASSOS, P. E. P. F. Hidrologia e biomassa primária do fitoplâncton no estuário do rio Capibaribe, Recife-Pernambuco. Recife, 1991, 187p. Dissertação (Mestrado em Oceanografia Biológica). Universidade Federal de Pernambuco, 1991.

TUNDISI, J. G. Produção primária standing-stock e fracionamento do fitoplâncton na laguna de Cananeia (SP). São Paulo. Tese (Doutorado em Ciências) Faculdade em Filosofia , Ciências e Letras, Universidade de São Paulo. $130 \mathrm{p}, 1969$.

UNESCO. International Oceanographic Tables. Wormly, 1973, v.2, 141 p.

UNESCO. Determination of photosynthethic pigmens in sea water, Report of SCOR/UNESCO Working group 17 with meat from 4 to 6 june 1964, Paris, 1966, 69 p. (Monographys on Oceanology Methodology).

VARELA. R., MASSA, I. Concentracion de clorofila $a$, feopigmentos y matéria en suspension en laguna de Raya, Isla de Margarita, Venezuela. Fundacion La Salle de ciências Naturales, estacion de Investigaciones marinas de margarita. Venezuela, n.105, p. 39-64, 1981. 


\section{ANEXOS}

Tabela 1 - Dados climatológicos do ano de 1998 (fornecido pela Estação Meteorológica de Itapirema Goiana, PE).

\begin{tabular}{l|c|c|c|c|c|c|c}
\hline \multicolumn{1}{|c|}{ Variáveis } & \multicolumn{3}{|c|}{ Temperatura $\left({ }^{\circ} \mathrm{C}\right)$} & \multicolumn{2}{c|}{ Umidade relativa (\%) } & $\begin{array}{c}\text { Precipitaçãó } \\
\text { Pluviométrica }\end{array}$ & $\begin{array}{c}\text { Insolação } \\
\text { total }\end{array}$ \\
\hline \multicolumn{1}{c|}{ Mês/98 } & Mínima & Máxima & Média & $9 \mathrm{~h}$ & $15 \mathrm{~h}$ & $(\mathrm{~mm})$ & $(\mathrm{h})$ \\
\hline Fevereiro & 23,9 & 30,2 & 27,0 & 74 & 80 & 26,7 & 285,4 \\
Abril & 23,5 & 29,8 & 26,6 & 80 & 79 & 106,2 & 272,2 \\
Junho & 21,7 & 29,2 & 25,5 & 84 & 85 & 90,9 & 238,7 \\
Agosto & 20,9 & 28,8 & 24,8 & 87 & 82 & 239,1 & $\ldots$ \\
Outubro & 21,6 & $\ldots$ & $\ldots$ & 75 & 77 & 48,8 & 203,3 \\
Dezembro & 22,2 & 30,5 & 26,3 & 71 & 70 & 19,4 & 310,3 \\
\hline \multicolumn{1}{c|}{ Média } & 22,3 & 29,7 & 26,0 & 78,5 & 78,8 & 88,5 & 216,9 \\
\hline
\end{tabular}

Tabela 2 -Parâmetros hidrológicos da estação 1 e 2 em diferentes regimes de maré, na praia de Carne de Vaca - Goiana, Pernambuco.

\begin{tabular}{|c|c|c|c|c|c|c|c|c|}
\hline \multicolumn{9}{|c|}{ ESTAÇÃO 1} \\
\hline PARÁMETROS & \multicolumn{2}{|c|}{ Salinidade $(\% o)$} & \multicolumn{2}{|c|}{ O.D $\left(\mathrm{ml} . \mathrm{L}^{-1}\right)$} & \multicolumn{2}{|c|}{ O.D. (\%) } & \multicolumn{2}{|c|}{ DBO (mg.L $\left.{ }^{-1}\right)$} \\
\hline Meses / maré & $\mathrm{BM}$ & $\mathrm{PM}$ & $\mathrm{BM}$ & PM & $\mathrm{BM}$ & $\mathrm{PM}$ & $\mathrm{BM}$ & PM \\
\hline Fevereiro & 36,74 & 36,19 & 4,41 & 4,24 & 102,08 & 100,95 & 0,73 & 0,17 \\
\hline Abril & 32,00 & 32,00 & 4,59 & 4,38 & 102,00 & 102,82 & & ... \\
\hline Junho & 35,58 & 32,92 & 4,48 & 3,44 & 100,90 & 71,22 & 0,37 & 0,30 \\
\hline Agosto & 26,82 & 21,51 & 4,94 & 6,06 & 101,64 & 126,25 & 0,56 & 1,38 \\
\hline Outubro & 18,96 & 18,84 & 5,95 & 5,01 & 121,18 & 107,05 & 0,12 & 0,46 \\
\hline Dezembro & 36,62 & 37,68 & 5,15 & 4,71 & 117,31 & 112,14 & 0,23 & 0,81 \\
\hline \multicolumn{9}{|c|}{ ESTAÇĀO 2} \\
\hline PARÂMETROS & \multicolumn{2}{|c|}{ Salinidade $(\% o)$} & \multicolumn{2}{|c|}{ O.D $\left(\mathrm{ml} . \mathrm{L}^{-1}\right)$} & \multicolumn{2}{|c|}{ O.D. (\%) } & \multicolumn{2}{|c|}{ DBO $\left(\mathrm{mg} \cdot \mathrm{L}^{-1}\right)$} \\
\hline Meses / maré & $\mathrm{BM}$ & PM & $\overline{\mathrm{BM}}$ & PM & $\overline{\mathrm{BM}}$ & PM & $\mathrm{BM}$ & $\mathrm{PM}$ \\
\hline Fevereiro & 36,19 & 36,07 & 4,62 & 5,67 & 107,44 & 135,00 & 0,65 & 0,18 \\
\hline Abril & 32,00 & 35,00 & 4,69 & 4,48 & 102,62 & 115,51 & & \\
\hline Junho & 33,99 & 34,25 & 3,86 & 2,40 & 86,16 & 50,83 & 0,34 & 3,42 \\
\hline Agosto & 32,39 & 32,92 & 4,94 & 5,28 & 102,06 & 123,94 & 0,91 & 7,28 \\
\hline Outubro & 34,78 & 35,85 & 5,95 & 5,01 & 108,85 & 97,81 & 1,47 & 2,10 \\
\hline Dezembro & 36,89 & 37,95 & 5,52 & 5,85 & 101,57 & 139,62 & 0,10 & 2,59 \\
\hline
\end{tabular}

$\mathrm{BM}=$ Baixa-mar

$\mathrm{PM}=$ Preamar 
Tabela 3 -Dados hidrológicos das estações 1 e 2 em diferentes regimes de maré, na praia de Carne de Vaca, Goiana, Pernambuco

\begin{tabular}{|c|c|c|c|c|c|c|c|c|c|c|}
\hline \multicolumn{11}{|c|}{$\begin{array}{l}\text { ESTAÇÃO } 1 \\
\end{array}$} \\
\hline PARÂMETROS & \multicolumn{2}{|c|}{$\mathrm{pH}$} & \multicolumn{2}{|c|}{$\begin{array}{c}{\mathrm{N}-\mathrm{NO}_{2}} \\
\left(\mu \mathrm{moles}^{-1} \mathrm{~L}^{-1}\right)\end{array}$} & \multicolumn{2}{|c|}{$\begin{array}{c}\mathrm{N}-\mathrm{NO}_{3} \\
\left(\mu \text { moles. } \mathrm{L}^{-1}\right)\end{array}$} & \multicolumn{2}{|c|}{$\begin{array}{c}{\mathrm{P}-\mathrm{PO}_{4}}_{4} \\
\left(\mu \text { moles.L }^{-1}\right)\end{array}$} & \multicolumn{2}{|c|}{$\mathrm{SI}-\mathrm{SIO}_{2}\left(\mu\right.$ moles.L $\left.\mathrm{L}^{-1}\right)$} \\
\hline Meses / maré & BM & PM & BM & $\mathrm{PM}$ & BM & $\mathrm{PM}$ & BM & PM & $\overline{\mathrm{BM}}$ & $\overline{\mathrm{PM}}$ \\
\hline Fevereiro & 8,38 & 8,45 & 0,246 & 0,092 & 0,454 & 0,696 & 0,233 & 0,341 & 18,253 & 10,774 \\
\hline Abril & 7,92 & 8,09 & 0,123 & 0,103 & 0,686 & 0,909 & 0,441 & 0,347 & 17,112 & 15,845 \\
\hline Junho & 8,33 & 8,31 & 0,001 & 0,031 & 0,641 & 0,728 & 0,216 & 0,424 & 9,211 & 27,379 \\
\hline Agosto & 8,35 & 8,47 & 0,102 & 0,328 & 0,708 & 1,857 & 0,229 & 0,341 & 45,422 & 76,139 \\
\hline Outubro & 7,87 & 7,89 & 0,010 & 0,010 & 1,756 & 0,812 & 0,249 & 0,001 & 1,479 & 1,056 \\
\hline Dezembro & 7,85 & 7,97 & 0,030 & 0,030 & 0,474 & 0,817 & 0,408 & 0,383 & 1,479 & 1,352 \\
\hline \multicolumn{11}{|c|}{ ESTAÇÃO 2} \\
\hline PARÂMETROS & \multicolumn{2}{|c|}{$\mathrm{pH}$} & \multicolumn{2}{|c|}{$\begin{array}{c}\mathrm{N}-\mathrm{NO}_{2} \\
\left(\mu \text { moles.L } \mathrm{L}^{-1}\right)\end{array}$} & \multicolumn{2}{|c|}{$\begin{array}{c}\mathrm{N}-\mathrm{NO}_{3} \\
\left(\mu \text { moles. } \mathrm{L}^{-1}\right)\end{array}$} & \multicolumn{2}{|c|}{$\begin{array}{c}\mathrm{P}-\mathrm{PO}_{4} \\
\left(\mu \mathrm{moles} . \mathrm{L}^{-1}\right)\end{array}$} & \multicolumn{2}{|c|}{ SI-SIO ${ }_{2}\left(\mu\right.$ moles.L $\left.L^{-1}\right)$} \\
\hline Meses / maré & $\mathrm{BM}$ & PM & BM & $\mathrm{PM}$ & BM & PM & BM & PM & $\mathrm{BM}$ & $\mathrm{PM}$ \\
\hline Fevereiro & 8,35 & 8,50 & 0,061 & 0,061 & 0,847 & 0,718 & 0,220 & 0,252 & 57,802 & 9,845 \\
\hline Abril & 8,00 & 8,39 & 0,070 & 0,072 & 0,969 & 0,617 & 0,241 & 0,354 & 15,676 & 25,985 \\
\hline Junho & 8,38 & 8,30 & 0,001 & 0,072 & 0,772 & 0,804 & 0,204 & 0,678 & 16,521 & 7,267 \\
\hline Agosto & 8,36 & 8,33 & 0,001 & 0,031 & 0,570 & 0,567 & 0,062 & 0,545 & 10,943 & 25,521 \\
\hline Outubro & 8,10 & 7,96 & 0,001 & 0,001 & 0,125 & 0,760 & 0,183 & 0,066 & 1,313 & 1,014 \\
\hline Dezembro & 7,90 & 7,93 & 0,001 & 0,001 & 0,804 & 1,453 & 0,175 & 0,225 & 1,225 & 1,083 \\
\hline
\end{tabular}

BM = Baixa-mar

$\mathrm{PM}=$ Preamar 